\title{
Correlation lengths of nonperturbative stochastic Yang-Mills fields
}

\author{
Dmitri Antonov* \\ Departamento de Física and Centro de Física das Interacções Fundamentais, \\ Instituto Superior Técnico, UT Lisboa, \\ Av. Rovisco Pais, 1049-001 Lisboa, Portugal \\ E-mail: dima@cfif.ist.utl.pt

\section{José Emílio F.T. Ribeiro} \\ Departamento de Física and Centro de Física das Interacções Fundamentais, \\ Instituto Superior Técnico, UT Lisboa, \\ Av. Rovisco Pais, 1049-001 Lisboa, Portugal \\ E-mail: emilioribeirodist.utl.pt
}

In the first part of this talk, we discuss the calculation of the correlation lengths of confining as well as of nonperturbative-nonconfining stochastic background Yang-Mills fields. This calculation was done in Ref. [1] by means of a direct analytic path-integral evaluation of the Green functions of the so-called one- and two-gluon gluelumps. Numerically, the obtained correlation lengths turn out to be in a good agreement with the results corresponding to the masses of these objects found on the lattice. They also agree with the earlier analytic results obtained within the Hamiltonian formalism. In the second part of the talk, we discuss the recent calculation of the quark condensate for various heavy flavors. This calculation was done in Ref. [2] by means of the effective-action formalism combined with the most general parametrization of the quark Wilson loop provided by the stochastic vacuum model. In particular, we show that the conventional formula for the heavy-quark condensate becomes inapplicable in the $c$-quark case, where it acquires the corrections that can reach up to 50\%. This conclusion agrees with the one drawn in Ref. [3], where the heavy-quark condensate was calculated by other analytic nonperturbative methods.

Xth Quark Confinement and the Hadron Spectrum

8-12 October 2012

TUM Campus Garching, Munich, Germany

\footnotetext{
* Speaker.
} 


\section{Introduction}

This talk addresses two fundamentally important issues of non-perturbative QCD. The first issue is the theoretical foundations of the stochastic model of the QCD vacuum [1], the second issue is the calculation of the quark condensate in terms of the gluon condensate for various heavy flavors [2]. We find it possible to combine these two subjects into a single talk, since we use for their study one and the same approach based on the analytic calculations of path integrals with the minimal-area surfaces. The two subjects also have a common starting point, which is the formula expressing the Wilson loop

$$
\langle W(C)\rangle \equiv\left\langle W\left[z_{\mu}\right]\right\rangle=\left\langle\operatorname{tr} \mathscr{P} \exp \left(i g \int_{0}^{s} d \tau T^{a} A_{\mu}^{a} \dot{z}_{\mu}\right)\right\rangle
$$

through the two-point correlation function of gluonic field strengths [4]:

$$
\langle W(C)\rangle \simeq \operatorname{tr} \exp \left[-\frac{g^{2}}{8} \int_{\Sigma_{\min }(C)} d \sigma_{\mu v}(x) \int_{\Sigma_{\min }(C)} d \sigma_{\lambda \rho}\left(x^{\prime}\right)\left\langle F_{\mu v}^{a}(x) T^{a} \Phi_{x x^{\prime}} F_{\lambda \rho}^{b}\left(x^{\prime}\right) T^{b} \Phi_{x^{\prime} x}\right\rangle\right] .
$$

Here, $F_{\mu \nu}^{a}=\partial_{\mu} A_{v}^{a}-\partial_{v} A_{\mu}^{a}+g f^{a b c} A_{\mu}^{b} A_{v}^{c}$ is the Yang-Mills field-strength tensor, and $T^{a}$ 's are the generators of the group $\mathrm{SU}\left(N_{c}\right)$ in the fundamental representation, obeying the commutation relation $\left[T^{a}, T^{b}\right]=i f^{a b c} T^{c}$. The Wilson loop is unambiguously defined by the minimal-area surface $\Sigma_{\min }(C)$ bounded by the contour $C$, which is parametrized by the vector-function $z_{\mu}(\tau)$. Furthermore, $\Phi_{x x^{\prime}} \equiv \mathscr{P} \exp \left[\operatorname{ig} \int_{x^{\prime}}^{x} d z_{\mu} A_{\mu}^{a}(z) T^{a}\right]$ denotes a phase factor along the straight-line path interconnecting the points $x^{\prime}$ and $x$, and the average $\langle\ldots\rangle$ is taken with respect to the Yang-Mills action $\frac{1}{4} \int d^{4} x\left(F_{\mu \nu}^{a}\right)^{2}$. The most general parametrization of the two-point correlation function, which is provided by the stochastic vacuum model $[4,5]$, contains two mutually independent tensor structures, namely

$$
\begin{gathered}
\operatorname{tr}\left\langle F_{\mu v}^{a}(x) T^{a} F_{\lambda \rho}^{b}(0) T^{b}\right\rangle= \\
=\left(\delta_{\mu \lambda} \delta_{v \rho}-\delta_{\mu \rho} \delta_{v \lambda}\right) D(x)+\frac{1}{2}\left[\partial_{\mu}\left(x_{\lambda} \delta_{v \rho}-x_{\rho} \delta_{v \lambda}\right)+\partial_{v}\left(x_{\rho} \delta_{\mu \lambda}-x_{\lambda} \delta_{\mu \rho}\right)\right] D_{1}(x) .
\end{gathered}
$$

As can be checked upon the substitution of Eq. (1.3) into Eq. (1.2), the function $D(x)$ yields confinement, while the function $D_{1}(x)$ exists already in massive QED, yielding only Yukawa-type interactions. According to the lattice data [6,7], both functions $D(x)$ and $D_{1}(x)$ fall off exponentially, and their correlation lengths are equal to each other within the errors of the corresponding lattice calculations. The equality of the two correlation lengths has nevertheless been questioned by the phenomenological studies done in Refs. $[8,9]$. The results of these studies were indicating that the correlation length of the function $D(x)$ could be smaller than that of the function $D_{1}(x)$. These indications were confirmed by the subsequent studies performed within the methods of potential non-relativistic QCD [10] and the background perturbation theory [11]. There, the correlation function (1.3) has been expressed via the Green functions of the so-called gluelumps. The latter are the bound states of gluons in the field of a hypothetical infinitely heavy source transforming under the adjoint representation. In particular, it has been found that the correlation lengths of the functions $D(x)$ and $D_{1}(x)$ are given by the inverse masses of, respectively, two- and one-gluon gluelumps, which are known from the lattice data (cf. Ref. [12]). Hence, in Section 2, we summarize the results of Ref. [1], where the analytic calculation of the gluelumps' Green functions has been shown to provide two different vacuum correlation lengths. 
In Section 3, we discuss the calculation of the heavy-quark condensate for various heavy flavors, which was done in Ref. [2]. To this end, we substitute the Wilson loop in the form given by Eqs. (1.2) and (1.3) into the following one-loop effective action [13, 14, 15]:

$$
\begin{gathered}
\left\langle\Gamma\left[A_{\mu}^{a}\right]\right\rangle=2 \int_{0}^{\infty} \frac{d s}{s} \mathrm{e}^{-M^{2} s} \times \\
\times \int_{P} \mathscr{D} z_{\mu} \int_{A} \mathscr{D} \psi_{\mu} \mathrm{e}^{-\int_{0}^{s} d \tau\left(\frac{1}{4} \dot{z}_{\mu}^{2}+\frac{1}{2} \psi_{\mu} \psi_{\mu}\right)} \exp \left[-2 \int_{0}^{s} d \tau \psi_{\mu} \psi_{v} \frac{\delta}{\delta \sigma_{\mu v}(z)}\right]\left\langle W\left[z_{\mu}\right]\right\rangle .
\end{gathered}
$$

Here, $P$ and $A$ stand, respectively, for the periodic and the antiperiodic boundary conditions, so that $\int_{P} \equiv \int_{z_{\mu}(s)=z_{\mu}(0)}, \int_{A} \equiv \int_{\psi_{\mu}(s)=-\psi_{\mu}(0)}$, and $M$ is the current quark mass. The corresponding expression for the heavy-quark condensate reads $\langle\bar{\psi} \psi\rangle=-\frac{1}{V} \frac{\partial}{\partial M}\left\langle\Gamma\left[A_{\mu}^{a}\right]\right\rangle$, where $V$ is the Euclidean four-volume occupied by the system. Equation (1.4) also uses the fact that the product $F_{\mu \nu}^{a} T^{a}$, which enters the quark spin term in the world-line action, can be recovered by means of the areaderivative operator $\frac{\delta}{\delta \sigma_{\mu \nu}}$ acting on the Wilson loop [16]. By virtue of this fact, all the gaugefield dependence of the effective action becomes encoded in the Wilson loop (1.1). Furthermore, since the Wilson loop is a color-neutral object, which is completely determined by the geometric characteristics of its contour, the problem at issue reduces to the calculation of the effective action in an auxiliary Abelian gauge field [14].

For the mean sizes of the Euclidean trajectory of a heavy quark smaller than the vacuum correlation length, one can use constant $F_{\mu \nu}^{a}$ as a leading approximation. By virtue of Eq. (1.2), this results in the following expression for the nonperturbative contribution to a small-sized Wilson loop (1.1) (cf. Ref. [4]):

$$
\langle W(C)\rangle=N_{c} \cdot \mathrm{e}^{-\frac{\left\langle\left(g F_{M v}^{a}\right)^{2}\right\rangle}{48 N_{C}} S_{\min }^{2}}
$$

Here, $\left\langle\left(g F_{\mu \nu}^{a}\right)^{2}\right\rangle$ is the gluon condensate, and $S_{\min }$ is the area of the surface $\Sigma_{\min }(C)$. As was shown in Ref. [14], Eq. (1.5), once substituted into Eq. (1.4), correctly reproduces the heavy-quark condensate obtained within the method of the SVZ sum rules [17]:

$$
\langle\bar{\psi} \psi\rangle_{\mathrm{SVZ}}=-\frac{\left\langle\left(g F_{\mu \nu}^{a}\right)^{2}\right\rangle}{48 \pi^{2} M}
$$

In Ref. [2] and in Section 3 below, we address the accuracy of Eq. (1.6) for various heavy flavors. To this end, we calculate the effective action (1.4) by using for the Wilson loop the following general expression resulting from Eqs. (1.2) and (1.3):

$$
\langle W(C)\rangle=N_{c} \cdot \exp \left[-\frac{\left\langle\left(g F_{\mu \nu}^{a}\right)^{2}\right\rangle}{96 N_{c}} \int_{\Sigma_{\min }(C)} d \sigma_{\mu v}(x) \int_{\Sigma_{\min }(C)} d \sigma_{\mu v}\left(x^{\prime}\right) \mathrm{e}^{-\left|x-x^{\prime}\right| / T_{g}}\right] .
$$

Here $T_{g}$ is the correlation length of the function $D(x)$, and we have for simplicity assumed the purely exponential parametrization of this function at all distances. Furthermore, the contribution of the function $D_{1}(x)$ to Eq. (1.7) has been disregarded. The reason for that is provided by the corresponding explicit calculation done in Ref. [2]. This calculation shows that, for the same exponential parametrization of the function $D_{1}(x)$, the contributions of this function to the effective action cancel out.

Finally, in Section 4, we provide a summary of the results reported in this talk. 


\section{Vacuum correlation lengths from the gluelump Green functions}

In the Yang-Mills theory, gluelumps define the correlation lengths of the field-strengths' twopoint function in the same way as, in full QCD, physically existing heavy-light mesons define the correlation length of a nonlocal quark condensate $\left\langle\bar{\psi}(x) \Phi_{x x^{\prime}} \psi\left(x^{\prime}\right)\right\rangle$ (cf. Refs. [9, 10, 11]). Unlike the case of the fundamental representation, the adjoint representation allows for two different types of heavy-light objects - those with a single gluon, called one-gluon gluelumps, and those with two gluons, called two-gluon gluelumps. While the first case is similar to the above-mentioned nonlocal quark condensate, the second case is conceptually different, since it corresponds to two gluons connected together with the heavy source by three strings in the fundamental representation. To find the correlation lengths of the functions $D(x)$ and $D_{1}(x)$, we use the relations of these functions to the Green functions of, respectively, two- and one-gluon gluelumps. These relations, found in Ref. [11], read

$$
D(x) \propto G_{2 \mathrm{gl}}(x), \quad D_{1}(x) \propto-\frac{d G_{1 \mathrm{gl}}(x)}{d x^{2}} .
$$

We consider first the Green function of a one-gluon gluelump,

$$
G_{1 \mathrm{gl}}(x)=\int_{0}^{\infty} d s \int_{0}^{x} \mathscr{D} z_{\mu} \exp \left(-\int_{0}^{s} \frac{\dot{z}_{\mu}^{2}}{4} d \lambda-\sigma S_{\min }\right),
$$

where the minimal surface of the area $S_{\min }$ is swept out by the string connecting a gluon to the adjointly charged source. Accordingly, the string tension $\sigma$ is also the one in the adjoint representation. Furthermore, the source of the gauge field is assumed to be static, which means that it evolves only along the $x_{4}$-axis, i.e. $x=(\mathbf{0}, L)$. The path integral entering Eq. (2.1) has been calculated in Ref. [1] by majorating $S_{\min }=\int_{0}^{L} d \tau|\mathbf{z}(\tau)|$ through the Cauchy-Schwarz inequality: $S_{\min } \leq \sqrt{L \int_{0}^{L} d \tau \mathbf{z}^{2}}$. This way, the said path integral has been reduced to that of an harmonic oscillator of a variable frequency. The resulting Green function, $G_{1 \mathrm{gl}}(x) \simeq \frac{\sigma}{\sqrt{3 \pi^{3}}} \mathrm{e}^{-\sqrt{6 \sigma}|x|}$, yields the mass of the one-gluon gluelump equal to $\sqrt{6 \sigma} \simeq 1.6 \mathrm{GeV}$. Here, the string tension in the adjoint representation has been evaluated via the so-called Casimir-scaling hypothesis [18]. This hypothesis, supported both by lattice simulations [19] and analytic studies [5, 20], suggests proportionality of the string tension in a given representation of the group $\mathrm{SU}\left(N_{c}\right)$ to the quadratic Casimir operator of that representation. For the adjoint representation of the group $\operatorname{SU}(3)$, it yields $\sigma=\frac{9}{4} \sigma_{\mathrm{f}}$, where the standard phenomenological value of the string tension in the fundamental representation is $\sigma_{\mathrm{f}}=(440 \mathrm{MeV})^{2}$. We observe that the above-quoted value of the mass of the one-gluon gluelump turns out to be close to the value of $1.5 \mathrm{GeV}$, which was obtained in Ref. [11] from the Schrödinger equation with the linear potential, as well as to the value of $1.4 \mathrm{GeV}$ obtained on the lattice in Ref. [12].

In the case of a two-gluon gluelump, the minimal surface formed by two gluons and the static source has a triangular cross-section. The area of this surface can be parametrized as $S_{\min }=$ $\int_{0}^{L} d \tau(|\mathbf{z}|+|\overline{\mathbf{z}}|+|\mathbf{z}-\overline{\mathbf{z}}|)$, where $\mathbf{z}$ and $\overline{\mathbf{z}}$ are the spatial coordinates of the gluons. To majorate this area-functional, a yet another form of the Cauchy-Schwarz inequality is useful: $S_{\min } \leq$ $\sqrt{3} \int_{0}^{L} d \tau \sqrt{\mathbf{z}^{2}+\overline{\mathbf{z}}^{2}+(\mathbf{z}-\overline{\mathbf{z}})^{2}}$. One can further majorate this expression in the same way as it was done above for the one-gluon gluelump. Then the resulting path integral for $G_{2 \mathrm{gl}}(x)$ represents two 
mutually interacting harmonic oscillators of variable frequencies. To calculate this path integral, one needs to perform a simultaneous diagonalization of the kinetic and the potential energies of the two gluons, which is possible owing to the known fact that two positively definite quadratic forms can be diagonalized simultaneously. As a result, one arrives at the path integral of two noninteracting harmonic oscillators, so that the path integration can be done analytically. Referring the reader for details to Ref. [1], we quote here the resulting mass of the two-gluon gluelump. It reads $6 \sqrt{\sigma_{\mathrm{f}}} \simeq 2.6 \mathrm{GeV}$, which is very close to the value of $2.56 \mathrm{GeV}$ found in Ref. [11] via the Hamiltonian approach.

\section{Quark condensate for various heavy flavors}

In order to calculate the effective action (1.4) with the Wilson loop (1.7), we choose the surface element $d \sigma_{\mu \nu}$ in the form of an oriented, infinitely thin triangle built up of the position vector $z_{\mu}(\tau)$ and the differential element $d z_{\mu}=\dot{z}_{\mu} d \tau$, namely $d \sigma_{\mu v}(z)=\frac{1}{2}\left(z_{\mu} \dot{z}_{v}-z_{v} \dot{z}_{\mu}\right) d \tau$. Then the Wilson loop can be represented in the form of a functional integral over an auxiliary antisymmetric-tensor field $B_{\mu v}$ :

$$
\langle W(C)\rangle=N_{c} \int\left[\prod_{\mu<v} \mathscr{D} B_{\mu \nu} \mathrm{e}^{-\frac{N_{c} T_{g}}{\pi^{2}\left\langle\left(g F_{\mu \nu}^{a}\right)^{2}\right\rangle} \int d^{4} x B_{\mu v}\left(-\partial^{2}+T_{g}^{-2}\right)^{5 / 2} B_{\mu \nu}}\right] \mathrm{e}^{\frac{i}{2} \int_{0}^{s} d \tau B_{\mu \nu}(z) z_{\mu} \dot{z}_{\nu}} .
$$

One recognizes in the latter exponential factor a Wilson loop corresponding to the Abelian gauge field $A_{v}(x)=\frac{1}{2} x_{\mu} B_{\mu v}(x)$. For this reason, one can write down for the effective action (1.4) a closed-form expression containing two strength tensors $F_{\mu \nu}=\partial_{\mu} A_{\nu}-\partial_{v} A_{\mu}$ of the field $A_{\mu}$. This expression reads

$$
\left\langle\Gamma\left[A_{\mu}^{a}\right]\right\rangle=2 N_{c} \int_{0}^{\infty} \frac{d s}{s} \frac{\mathrm{e}^{-M^{2} s}}{(4 \pi)^{2}}\left\langle\int d^{4} x F_{\mu v}(x) \mathrm{F}(\xi) F_{\mu v}(x)\right\rangle_{B},
$$

where the $B$-average is defined by the functional integral in Eq. (3.1), and $\mathrm{F}(\xi)$ is the following formfactor [15]: $\mathrm{F}(\xi)=\frac{f(\xi)-1}{2 \xi}-\frac{1}{4} f(\xi)$. In this formula, $f(\xi)=\int_{0}^{1} d u \mathrm{e}^{u(1-u) \xi}$, and $\xi=s D_{\mu}^{2}$, where $D_{\mu}=\partial_{\mu}-i A_{\mu}$ is the covariant derivative corresponding to the auxiliary Abelian field $A_{\mu}$. Representing further the formfactor $F(\xi)$ in an equivalent form,

$$
\mathrm{F}(\xi)=\frac{1}{2(4 \pi s)^{2}} \int_{0}^{1} d u \int d^{4} y\left(\frac{4 s}{y^{2}}-\frac{1}{2[u(1-u)]^{2}}\right) \mathrm{e}^{-\frac{y^{2}}{4 u(1-u) s}+y_{\mu} D_{\mu}}
$$

and using the equality $\left\langle F_{\mu v}(x) \mathrm{e}^{y_{\mu} D_{\mu}} F_{\mu v}(x)\right\rangle_{B}=\left\langle F_{\mu v}(0) F_{\mu v}(y)\right\rangle_{B}$, we see that the $B$-average yields expressions proportional to $\left\langle\left(g F_{\mu \nu}^{a}\right)^{2}\right\rangle \mathrm{e}^{-|y| / T_{g}}$. This fact allows us to represent the quark condensate in the form of a product of the gluon condensate and a certain function of the current quark mass. Denoting $\lambda \equiv M T_{g}$, we obtain the following ratio $I(\lambda) \equiv \frac{\langle\bar{\psi} \psi\rangle}{\langle\bar{\psi} \psi\rangle_{\mathrm{SVZ}}}$ of the quark condensate thus obtained to the one given by Eq. (1.6):

$$
\begin{gathered}
I(\lambda)=\frac{3 \lambda^{2}}{4} \int_{0}^{1} \frac{d u}{1-a^{2}}\left\{4+\left(\frac{a}{\lambda}\right)^{2} \cdot \frac{2 a^{2}+1}{1-a^{2}}+\frac{3}{a^{2}-1}-\left(\frac{a}{2 \lambda}\right)^{2} \cdot \frac{13 a^{2}+2}{\left(a^{2}-1\right)^{2}}+\right. \\
\left.+\frac{\arccos (1 / a)}{\left(a^{2}-1\right)^{3 / 2}}\left[\frac{3 a^{4}}{\lambda^{2}}-5 a^{2}+2+\left(\frac{a}{2 \lambda}\right)^{2} \cdot \frac{3 a^{2}\left(a^{2}+4\right)}{a^{2}-1}\right]\right\}, \quad \text { where } a \equiv \frac{\lambda}{\sqrt{u(1-u)}} .
\end{gathered}
$$


The remaining $u$-integral in this expression has been calculated numerically. Using the value of $T_{g} \simeq 0.34 \mathrm{fm}$ in full QCD with light flavors [7], as well as the standard values of the quark masses, $M_{c} \simeq 1.3 \mathrm{GeV}, M_{b} \simeq 4.2 \mathrm{GeV}$, and $M_{t} \simeq 173 \mathrm{GeV}$, we obtain [2]:

$$
I\left(M_{c} T_{g}\right) \simeq 0.60, \quad I\left(M_{b} T_{g}\right) \simeq 0.84, \quad I\left(M_{t} T_{g}\right) \simeq 0.996 .
$$

The obtained decrease of $I(\lambda)$ with the decrease of $M$ shows that, if one keeps using Eq. (1.6) while decreasing $M$, then $M$ in Eq. (1.6) effectively gets larger than just the current quark mass.

Lattice calculations done in the SU(3) Yang-Mills theory [6], as well as the results of analytic studies $[1,9,10,11]$ (cf. also Section 2), suggest the values of $T_{g}$ in that theory which can be smaller than the above-quoted one by a factor of $2 \div 3$. Accordingly, in QCD with only heavy flavors, the value of $T_{g}$ should also be smaller than the one in full QCD with light flavors. This observation suggests that the corrections to Eq. (1.6) can be even larger than those given by Eq. (3.4). As a result, Eq. (1.6) becomes inapplicable to the $c$-quark, since the corresponding $40 \%$-correction given by Eq. (3.4) can increase further, up to $53 \%$ (cf. Ref. [2]). We notice that a qualitatively similar conclusion has been drawn in Ref. [3], where the leading correction to Eq. (1.6) has been evaluated through a non-perturbative gluon propagator in the Fock-Schwinger gauge.

Notice finally that, while the adopted world-line formalism has an advantage of being gaugeinvariant, it fails to calculate the self-energy contribution to the dynamical constituent quark mass. It is for this reason that we had to restrict our analysis to the case of heavy quarks, where this contribution can be safely disregarded compared to the current quark mass. In particular, one can use $1 / T_{g}$ as a lower limit for the continuously varied heavy-quark mass parameter $M$, noticing that the current masses of $u-, d$-, and $s$-quarks are indeed smaller than $1 / T_{g}$. One then finds from Eq. (3.3) that the absolute value of the quark condensate for $M=\frac{1}{T_{g}}$ is by $64 \%$ smaller than the absolute value corresponding to Eq. (1.6).

\section{Summary}

In the first part of this talk, we have summarized the results of Ref. [1], which demonstrate that the confining and the nonconfining nonperturbative self-interactions of stochastic background Yang-Mills fields can have different correlation lengths. In Ref. [1], these lengths have been obtained from the Green functions of the one- and the two-gluon gluelumps. This has been done by means of an analytic calculation of the corresponding quantum-mechanical path integrals for one and two gluons connected by strings with the static source and with each other. In agreement with the previous studies $[8,9,10,11]$, the correlation function $D(x)$, which describes confining interactions of the background fields, turns out to have a smaller correlation length than the function $D_{1}(x)$, which describes nonconfining nonperturbative interactions. Quantitatively, the inverse correlation length of the function $D(x)$, defined by the mass of the two-gluon gluelump, was found to be $\simeq 2.6 \mathrm{GeV}$, while the inverse correlation length of the function $D_{1}(x)$, defined by the mass of the one-gluon gluelump, is $\simeq 1.6 \mathrm{GeV}$. These results are very close to the corresponding values of $2.56 \mathrm{GeV}$ and $1.5 \mathrm{GeV}$, which were obtained by using the Hamiltonian methods [11] and on the lattice [12].

In the second part of the talk, we have summarized the calculation of the heavy-quark condensate done in Ref. [2]. This condensate has been obtained for various heavy flavors from the 
one-loop effective action (1.4) by using for the latter the closed-form expression (3.2) found in Ref. [15]. This approach allowed us to use for the quark Wilson loop the most general ansatz (1.7), which is provided by the stochastic vacuum model [4, 5]. Equations (3.4) provide the ratios of the quark condensate thus obtained to the standard expression (1.6) for the heavy-quark condensate. As one can see, corrections to Eq. (1.6) amount to $16 \%$ for the $b$-quark, and vary from $40 \%$ to $53 \%$ for the $c$-quark (cf. Ref. [2]), making Eq. (1.6) inapplicable in the latter case. As has also been found in Ref. [2], for the most simple, exponential, parametrization of the two-point correlation function of gluonic field strengths, the value of the heavy-quark condensate is not affected by nonconfining nonperturbative interactions of gluonic fields, which are described by the function $D_{1}(x)$.

The latter result parallels the proportionality of the quark condensate in the chiral limit to only the chromo-electric part of the full gluon condensate,

$$
\langle\bar{\psi} \psi\rangle_{\text {chiral }} \propto-T_{g}\left\langle\left(g \mathbf{E}^{a}\right)^{2}\right\rangle,
$$

which was found in Ref. [21]. How Eq. (1.6), along with the corrections discussed above, goes over with the further decrease of the current quark mass to Eq. (4.1) remains an open problem. A related problem concerns a derivation of the constituent masses of light quarks within the same gaugeinvariant formalism of the effective action. In our opinion, these two problems deserve dedicated studies.

\section{Acknowledgments}

We thank the organizers of the conference 'Quark confinement and the hadron spectrum $X$ ' for an opportunity to present these results in a very nice and stimulating atmosphere. The work of D.A. was supported by the Portuguese Foundation for Science and Technology (FCT, program Ciência-2008) and by the Center for Physics of Fundamental Interactions (CFIF) at Instituto Superior Técnico (IST), Lisbon.

\section{References}

[1] D. Antonov, Phys. Lett. B 696, 214 (2011).

[2] D. Antonov and J. E. F. T. Ribeiro, Eur. Phys. J. C 72, 2179 (2012).

[3] V. Shevchenko and Yu. Simonov, Phys. Rev. D 65, 074029 (2002).

[4] H. G. Dosch and Yu. A. Simonov, Phys. Lett. B 205, 339 (1988).

[5] A. Di Giacomo, H. G. Dosch, V. I. Shevchenko and Yu. A. Simonov, Phys. Rept. 372, 319 (2002).

[6] A. Di Giacomo and H. Panagopoulos, Phys. Lett. B 285, 133 (1992).

[7] M. D’Elia, A. Di Giacomo and E. Meggiolaro, Phys. Lett. B 408, 315 (1997).

[8] G. S. Bali, N. Brambilla and A. Vairo, Phys. Lett. B 421, 265 (1998).

[9] H. G. Dosch, M. Eidemüller and M. Jamin, Phys. Lett. B 452, 379 (1999).

[10] N. Brambilla, A. Pineda, J. Soto and A. Vairo, Nucl. Phys. B 566, 275 (2000).

[11] Yu. A. Simonov, Nucl. Phys. B 592, 350 (2001); Phys. Atom. Nucl. 69, 528 (2006). 
[12] N. A. Campbell, I. H. Jorysz and C. Michael, Phys. Lett. B 167, 91 (1986); I. H. Jorysz and C. Michael, Nucl. Phys. B 302, 448 (1988); M. Foster et al. [UKQCD Collaboration], Phys. Rev. D 59, 094509 (1999).

[13] Z. Bern and D. A. Kosower, Phys. Rev. Lett. 66, 1669 (1991); Nucl. Phys. B 379, 451 (1992); M. J. Strassler, Nucl. Phys. B 385, 145 (1992); for reviews see: M. Reuter, M. G. Schmidt and C. Schubert, Annals Phys. 259, 313 (1997); C. Schubert, Phys. Rept. 355, 73 (2001).

[14] D. Antonov and J. E. F. T. Ribeiro, Phys. Rev. D 81, 054027 (2010).

[15] M. G. Schmidt and C. Schubert, Phys. Lett. B 318, 438 (1993); for the bosonic case see: A. O. Barvinsky and G. A. Vilkovisky, Nucl. Phys. B 333, 471 (1990).

[16] Yu. M. Makeenko and A. A. Migdal, Phys. Lett. B 88, 135 (1979); Nucl. Phys. B 188, 269 (1981).

[17] M. A. Shifman, A. I. Vainshtein and V. I. Zakharov, Nucl. Phys. B 147, 385 (1979); for a review see: S. Narison, QCD Spectral Sum Rules (World Scientific, Singapore, 1989).

[18] J. Ambjørn, P. Olesen and C. Peterson, Nucl. Phys. B 240, 189, 533 (1984).

[19] G. S. Bali, Phys. Rev. D 62, 114503 (2000).

[20] D. Antonov and L. Del Debbio, JHEP 12, 060 (2003); D. Antonov, L. Del Debbio and D. Ebert, JHEP 12, 022 (2004).

[21] N. Brambilla and A. Vairo, Phys. Lett. B 407, 167 (1997); P. Bicudo, N. Brambilla, J. E. F. T. Ribeiro and A. Vairo, Phys. Lett. B 442, 349 (1998). 